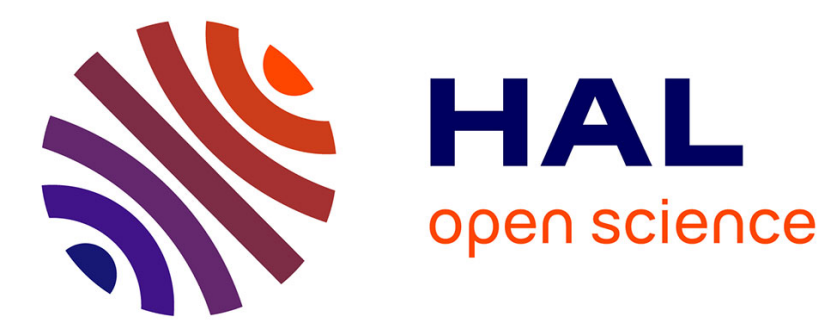

\title{
Frequency-domain, wide-field laser Doppler in vivo imaging
}

Michael Atlan, Michel Gross, Benoit C. Forget, Tania Vitalis, Armelle Rancillac, Andrew K. Dunn

\section{- To cite this version:}

Michael Atlan, Michel Gross, Benoit C. Forget, Tania Vitalis, Armelle Rancillac, et al.. Frequencydomain, wide-field laser Doppler in vivo imaging. Optics Letters, 2006, 31 (18), pp.2762-2764. hal00258697

\section{HAL Id: hal-00258697 \\ https://hal.science/hal-00258697}

Submitted on 25 Feb 2008

HAL is a multi-disciplinary open access archive for the deposit and dissemination of scientific research documents, whether they are published or not. The documents may come from teaching and research institutions in France or abroad, or from public or private research centers.
L'archive ouverte pluridisciplinaire HAL, est destinée au dépôt et à la diffusion de documents scientifiques de niveau recherche, publiés ou non, émanant des établissements d'enseignement et de recherche français ou étrangers, des laboratoires publics ou privés. 


\title{
Frequency-domain, wide-field laser Doppler in vivo imaging
}

\author{
M. Atlan, M. Gross, ${ }^{1}$ B. C. Forget,$^{2}$ T. Vitalis, A. Rancillac, ${ }^{3}$ and A. K. Dunn ${ }^{4}$ \\ ${ }^{1}$ Laboratoire Kastler-Brossel, UMR 8552 (ENS, CNRS, UMPC), Ecole Normale Supérieure, \\ 10 rue Lhomond F-75231 Paris cedex 05. France \\ ${ }^{2}$ Laboratoire d'Optique, École Supérieure de Physique et de Chimie Industrielles de la Ville de Paris, \\ CNRS UPR A0005, Université Pierre et Marie Curie, \\ 10 rue Vauquelin F-75231 Paris cedex 05. France \\ ${ }^{3}$ Laboratoire de Neurobiologie et Diversité Cellulaire, \\ École Supérieure de Physique et de Chimie Industrielles de la Ville de Paris, \\ CNRS UMR 7637, 10 rue Vauquelin F-75231 Paris cedex 05. France \\ ${ }^{4}$ University of Texas at Austin. Biomedical Engineering Department - ENS \\ 1 University Station, CO 800 Austin, TX 78712-0238. United States
}

\begin{abstract}
We present a new instrument, based on a low frame rate $(8 \mathrm{~Hz}) \mathrm{CCD}$ camera used in a heterodyne optical-mixing configuration, that can create wide-field laser Doppler maps. As an illustration, we show results obtained in a mouse brain, in vivo, showing the Doppler signature of blood flow. The instrument is based on the frequency-shifting digital holography scheme.
\end{abstract}

OCIS 170.3340170 .6480090 .2880

Imaging blood flow is an essential tool to assess many physiologic processes and diseases. Several instruments have been proposed to produce blood flow maps. These instruments can be grouped in two categories : those which are based on time domain measurements (laser Doppler techniques) and those based on spatial domain measurements (speckle contrast analysis techniques) $[1,2]$. The first Laser Doppler experiment is attributed to Yeh and Cummins [3], who measured the frequency shift of light scattered by moving particles with a spectrometer.

Recently, Serov et al. proposed [4] and designed [5, 6] a full-field Laser Doppler imaging technique based on the acquisition of a sequence of images by a fast CMOS detector. This method requires on the one hand a frame rate high enough to set the cut-off frequency of the restituable spectrum higher than the ones induced by typical blood flow, eg.[6] $\sim 20 \mathrm{kHz}$ and, on the other hand, a sequence of images large enough to reach a 50 $\mathrm{Hz}$ spectral resolution [5].

Laser speckle contrast analysis [7] has been proposed as an alternative to scanning laser Doppler imaging as a full-field imaging technique. In this technique, the first order spatial statistics of the speckle pattern are used to form blood flow images. It has been successfully used in applications including noninvasive skin capillary blood flow imaging [8] and functional brain imaging [1].

Laser Doppler and speckle contrast analysis turn out to be based on the same underlying phenomenon [2, 9-11]. A new experimental approach is proposed here, consisting of a frequency domain measurement onto a parallel detector. This measurement is based on an optical mixing scheme.

The setup consists in a heterodyne measurement of scattered (object) optical field $E_{O}$. The object is illuminated over $\mathrm{a} \sim 1 \times 1 \mathrm{~cm}^{2}$ area (the region to be imaged). The backscattered laser wave is mixed with a frequency-shifted local oscillator (LO) $E_{L O}$ field.

Laser light is provided by a $658 \mathrm{~nm}$ (angular frequency $\left.\omega_{L}\right), 80 \mathrm{~mW}$ CW Mitsubishi ML120G21 single-mode diode. Two Bragg cells (Acousto-optic Modulators, AOMs), are used as beam frequency shifters (see fig. 1). The LO angular frequency $\omega_{L O}$ is shifted by the difference $\Delta \omega_{A O M}$ in driving frequencies of the set of Bragg cells : $\omega_{L O}=\omega_{L}+\Delta \omega_{A O M}$. A 1.3 Megapixel PCO PixelFly camera (framerate $\omega_{S}$, square pixels, pixel size $6.7 \mu \mathrm{m}$ ) is used to record a sequence of $m$ images (namely $I_{k}$, where $k=1, \ldots, m$ ) of the interference pattern resulting from optical mixing between the LO and object fields. The detection plane is set $50 \mathrm{~cm}$ away from the object plane. A circular pupil set in front of the object limits the field of view. Since the spatial field of interest in the object is $\approx 1 \mathrm{~cm}^{2}$, it matches the natural heterodyne parallel detection image field, avoiding the need for any lens in the object path.

The optical configuration is a typical holographic setup, called lensless Fourier (off-axis) holography setup $[12,13]$, in which the point source of the spherical reference wave (LO focal point) is located in the plane of the object. The lensless Fourier setup is used to avoid the need of a general Fresnel reconstruction algorithm [14] to assess the spatial distribution of the scattered field in the object plane. In this case, the reconstruction algorithm consists of only one spatial fast Fourier transform [13] (FFT).

The digital hologram is recorded according to the frequency-shifting method introduced in the heterodyne holography technique, used in several imaging and detection schemes [15-18] and which turns out to be sensitive enough to discriminate Doppler-shifted 

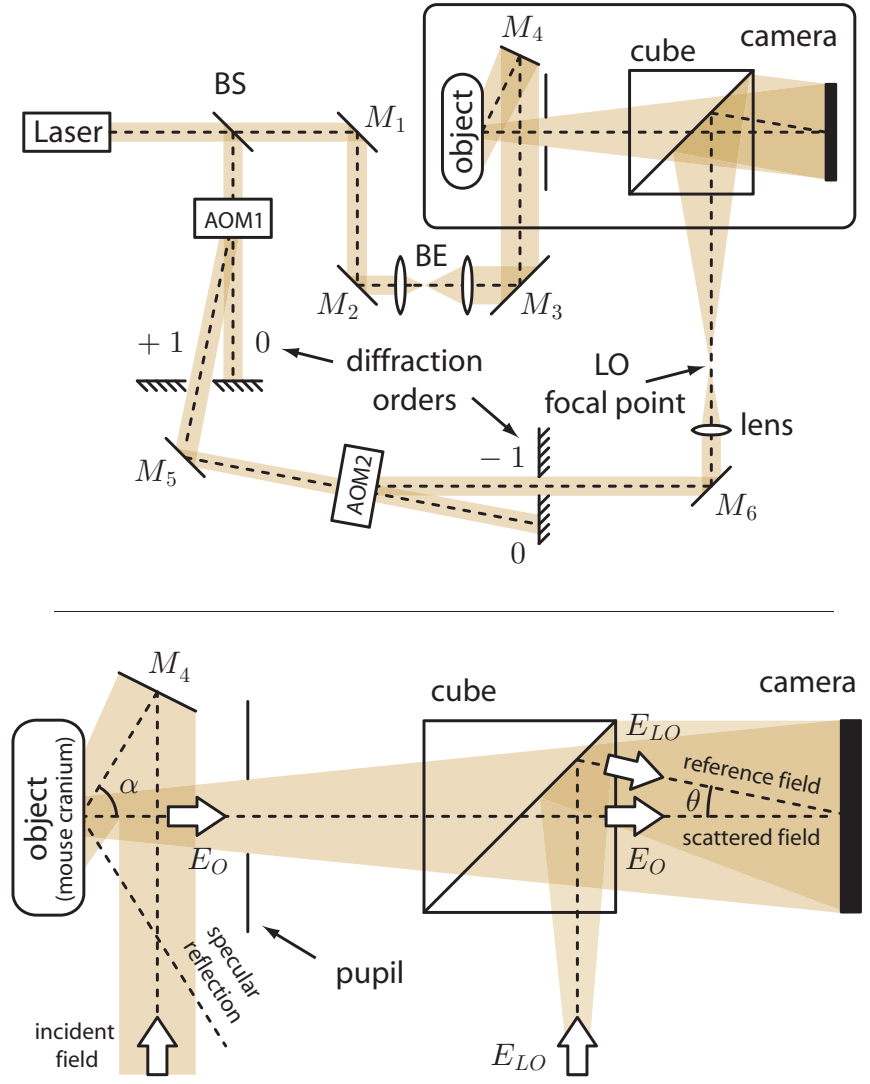

FIG. 1: Optical configuration (general view, upper fig. Inset, lower fig.). AOMs : Bragg cells. BS : beam splitter. BE : beam expander. $M_{1}$ to $M_{6}$ : mirors. $E_{O}$ : Object (scattered) field. $E_{L O}$ : reference (LO) field.

photons, according to their frequency, from background light $[16,18]$. The frequency-shifting method was chosen as an accurate [15] phase-shifting [19] detection method. To create a map of the $\Delta \omega$ angular frequency component (corresponding to the $\Delta f=\Delta \omega /(2 \pi)$ frequency, in $\mathrm{Hz}$ ) of the scattered field in the image plane, the LO frequency is set to $\omega_{L O}=\omega_{L}+\Delta \omega+\omega_{S} / n$, where the integer $n$ satisfies $n \geq 2$.

The power $S\left(\omega=\omega_{L}+\Delta \omega\right)$ of the $\Delta \omega$ frequency component of the measured object field is computed in parallel on the pixels of the image, according to the $n$-phase demodulation method $[16,17]$. It consists in calculating the squared amplitude of the $\omega_{S} / n \mathrm{AC}$ component of intensity fluctuations $I_{k}$ by a time-domain FFT: $S(\omega)=A\left|\sum_{k=1}^{n} I_{k} \exp (-2 j(k-1) \pi / n)\right|^{2}$, where $A$ is a constant and $j^{2}=-1$.

In the reported experiment, the cranium of the mouse is illuminated with an incidence angle $\alpha \simeq 60^{\circ}$. The angle between the object and LO arms is set to $\theta \simeq 1^{\circ}$. This experiment was designed to determine whether the instrument would allow detection of the cerebrocortical blood flow.
Animal procedures were conducted in strict compliance with approved institutional protocols and in accordance with the provisions for animal care and use described in the European Communities Council directive of $24^{\text {th }}$ November 1986 (86-16-09/EEC). Five C57/Bl6 mice (Charles River) aged P25-P30 were anaesthetised with a mixture of Xylazine-Ketamine $(1 \mathrm{mg} / \mathrm{kg} \mathrm{IP,} 10 \mathrm{mg} / \mathrm{kg}$ IP). Animals were positioned on a stereotaxic frame (World precision Instruments) to ensure stability of the preparation. Cranial skin and subcutaneous tissue were excised linearly over the sagittal suture and cortical bones were preserved.

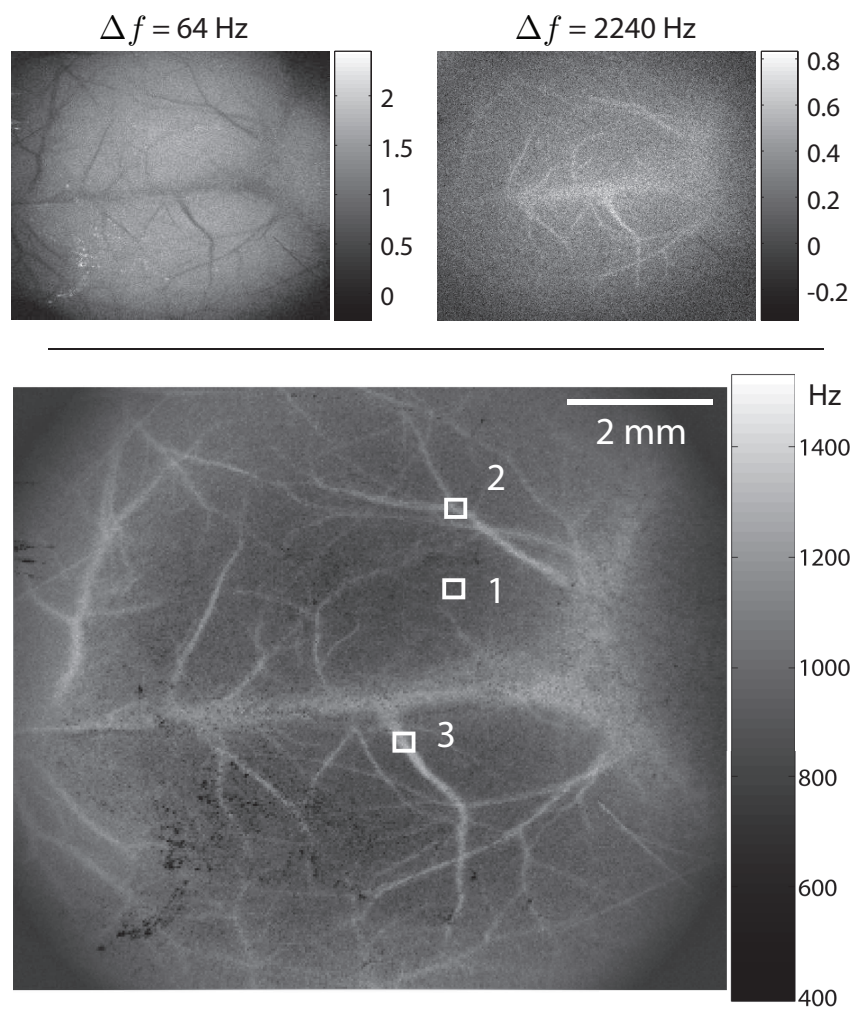

FIG. 2: Power maps of the $\Delta f=64 \mathrm{~Hz}$ and $\Delta f=2240 \mathrm{~Hz}$ frequency components of the scattered field (top, log. scale) and perfusion map, i.e. RMS frequency shift, in Hertz (bottom). The image shows a dorsal view of the mouse cranium (anterior on the left, posterior on the right). The superficial dorsal venous system and some of the superficial cerebral arteries are visible.

To assess bioflow [20], the root mean square (RMS) frequency shift $\left\langle\Delta \omega^{2}\right\rangle^{1 / 2}$ is calculated with respect to the $S(\omega)$ distribution measured at 80 linearly spaced frequency points in the $0 \rightarrow 2528 \mathrm{~Hz}$ range (spacing interval : $32 \mathrm{~Hz}$ ). The camera framerate was set to $8 \mathrm{~Hz}$. A sequence of $m=32$ images sampled during $32 / 8=4$ seconds was taken to build each $S\left(\omega=\omega_{L}+\Delta \omega\right)$ map at one given $\Delta \omega$ frequency point, setting $\Delta \omega_{A O M}=\Delta \omega+\omega_{S} / n$, where $n=4$. To increase SNR, $S(\omega)$ was averaged by 
means of an accumulation of sliding $n$-phase demodulation along the $m$ image sequence. The whole measurement took 5 minutes and 20 seconds $(=32$ images $\times$ 80 frequency shifts $\times 1 / 8$ second exposure time). Two $S(\omega)$ spatial maps at a low $(\Delta f=64 \mathrm{~Hz})$ and higher $(\Delta f=2240 \mathrm{~Hz})$ frequency shift and a spatial map of the RMS the frequency shift (in $\mathrm{Hz}$ ) are represented on fig. 2. Three spectra, averaged over $5 \times 5$ pixels re-

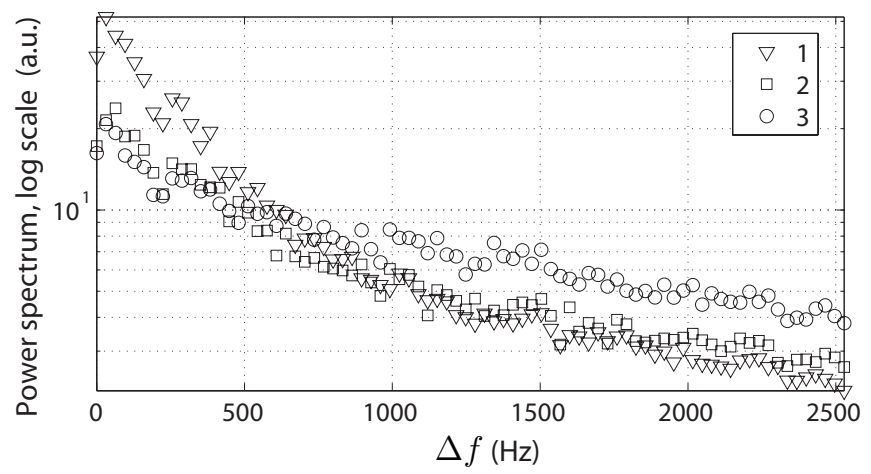

FIG. 3: Spectra calculated in the three $5 \times 5$ pixels ROI outlined in fig. 2 (log. scale).

gions of interest (ROI, outlined in fig. 2) are displayed in fig. 3. The larger blood vessels (eg. ROI 3) induce a broader spectrum than the smaller ones (ROI 2). In the parenchyma (ROI 1), spectra are narrower, suggesting a reduced blood perfusion. Hence the contrast reversal between the two spectral maps in fig. 2. An additional in vitro calibration experiment was done to assess the order of magnitude of the measured RMS the frequency shift for various flow rates. The result is shown in fig. 4; it demonstrates an approximately linear relationship between the average speed (from 0 to $750 \mu \mathrm{m} . \mathrm{s}^{-1}$ ) and the measured frequency shift.

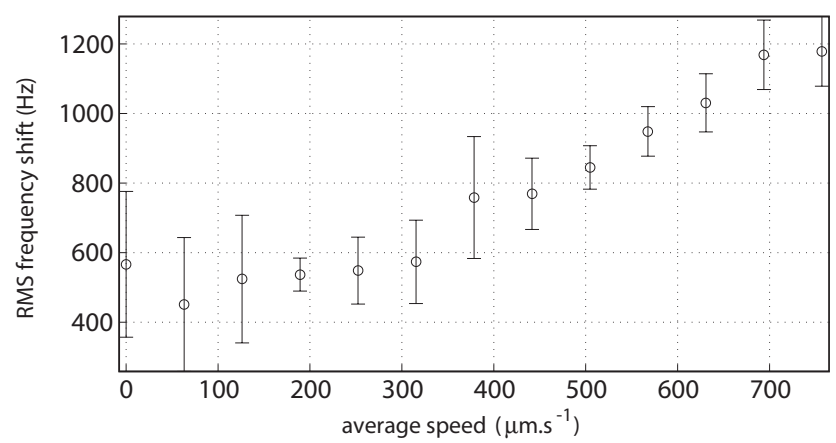

FIG. 4: In vitro measurement of the light scattered by a $510^{-3}$ vol. frac. suspension of $1 \mu \mathrm{m}$ latex beads flowing through a transparent tube of $580 \mu \mathrm{m}$ of diameter. Average RMS frequency shift vs. average speed of flow.

In conclusion, this scheme based on heterodyne optical mixing onto a parallel detector is particulary suited to wide-field laser Doppler imaging. An arbitrary frequency component of the object field can be acquired at once. The available range of frequency shifts at which measurements can be made is far more extended than in any of the concurrent techniques, since the measurement is directly performed in the frequency domain. The actual limit lies in the system used to shift the LO frequency. As an indication, the RF-driven AOMs combination can shift this frequency up to $10 \mathrm{Mhz}$.

The measurement is sensitive : thanks to the off-axis holographic setup, the low frequency noise components (homodyne intensity terms, resulting from laser instabilities and scattered light self interference) within the camera bandwidth are efficiently filtered-out [16, 17].

The Doppler map is not restituted over the total number of pixels of the camera. Only up to $1 / 4$ of the pixels of the frame can be used to describe the image, as a result of the off-axis holographic scheme. Nevertheless, both temporal and spatial resolution are potentially high. This combination is enabled by a measurement performed at one frequency point at a time.

A faster camera could be used to improve temporal resolution and still keep a spectral resolution acceptable for this measurement, since the spectral resolution is defined by the heterodyne bandwidth (inverse of the acquisition time). A detection array with a large number of pixels should be used to perform an efficient wide-field and high spatial resolution detection. Due to the data transfer rate limit encountered, the balance between the number of pixels and the frame rate of the detector should be guided by the application needs in terms of temporal, spectral and spatial resolution.

Contact : atlan@lkb.ens.fr

[1] A. Dunn, H. Bolay, M. A. Moskowitz, and D. A. Boas. Jounal of Cerebral Blood Flow and Metabolism, 21(3):195-201, 2001.

[2] T. Yoshimura. JOSA. A., 3:1032, 1986.

[3] Y. Yeh and H. Z. Cummins. Appl. Phys. Lett., 4:176-179, 1964.

[4] A. Serov, W. Steenbergen, and F. de Mul. Optics Letters, $27: 300,2002$.

[5] A. Serov, B. Steinacher, and T. Lasser. Opt. Ex., 13(10):3681, 2005.

[6] A. Serov and T. Lasser. Opt. Express, 13:6416-6428, 2005.

[7] A. F. Fercher and J. D. Briers. Opt. Commun., 37:326, 1981.

[8] J. David Briers, Glenn Richards, and Xiao Wei He. Journal of Biomedical Optics, 4(1):164-175, 1999.

[9] J. D. Briers. Physiological Measurement, 22:R35-R66, 2001.

[10] R. Bonner and R. Nossal. Applied Optics, 20:2097-2107, 1981.

[11] J. A. Briers. JOSA A., 13:345, 1996. 
[12] U. Schnars and W. P. O. Juptner. Meas. Sci. Technol., 13:R85-R101, 2002.

[13] Christoph Wagner, Sonke Seebacher, Wolfgang Osten, and Werner Juptner. Applied Optics, 38:4812-4820, 1999.

[14] U. Schnars. Journal of Optical Society of America A., 11(7):2011, 1994.

[15] F. LeClerc, L. Collot, and M. Gross. Optics Letters, 25(10):716-718, 2000

[16] M. Gross, P. Goy, B.C. Forget, M. Atlan, F. Ramaz, A.C.
Boccara, and A.K. Dunn. Opt. Lett., 30(11), 2005.

[17] M. Atlan, B.C. Forget, F. Ramaz, A.C. Boccara, and M. Gross. Opt. Lett., 30(11), 2005.

[18] M. Gross, P. Goy, and M. Al-Koussa. Optics Letters, 28:2482-2484, 2003.

[19] I. Yamaguchi and T. Zhang. Optics Letters, 18:31, 1997.

[20] P. Starukhin, S. Ulyanov, E. Galanzha, and V. Tuchin. Applied Optics, 39:2823, 2000. 